\title{
Parallel Distributed Detection for Wireless Sensor Networks:Performance Analysis and Design
}

\author{
Israfil Bahceci \\ School of Elect. \& Comp. Eng. \\ Georgia Institute of Technology \\ Atlanta, GA 30332, USA \\ Email: bahceci@ece.gatech.edu
}

\author{
Ghassan Al-Regib \\ School of Electr. \& Comp Eng. \\ Georgia Institute of Technology \\ Atlanta, GA 30332, USA \\ Email: gregib@ece.gatech.edu
}

\author{
Yucel Altunbasak \\ School of Elect. \& Comp. Eng. \\ Georgia Institute of Technology \\ Atlanta, GA 30332, USA \\ Email: yucel@ece.gatech.edu
}

\begin{abstract}
Parallel distributed detection for wireless sensor networks is studied in this paper. The network consists of a set of local sensors and a fusion center. Each local sensor makes a binary (single-bit) or M-ary (multi-bit) decision and passes it to the fusion center where a final decision is made. The links between the local sensors and the fusion center are subject to fading and additive noise resulting in corruption of the transmitted decisions. We analyze the performance of the decision fusion based on likelihood ratio tests and derive false alarm and detection probabilities. Based on the theoretical probability expressions, we design optimal decision rules for the local sensors and the fusion center. Finally, we illustrate the performance of the parallel fusion by numerical examples.
\end{abstract}

\section{INTRODUCTION}

Wireless sensor networks is an emerging technology that experiences a pervasive trend in many application areas including environment monitoring, health, security and surveillance, and robotic exploration [1]. Networks of sensor systems allow for many distributed processing and cooperative communication techniques [2-5]. In this paper, we focus on distributed detection that are specially tailored for wireless sensor networks [5]. In distributed detection, each sensor sends its observation to the fusion center where a global decision is made. Because of the bandwidth and energy limitations, instead of sending raw data to the fusion center, each sensor performs a local detection process and sends its decision (possibly consisting of a few bits) to the fusion center. The fusion center collects all decisions from all sensors and performs a final decision on the hypothesis under investigation.

In the literature, distributed detection has been considered for three major topologies: parallel, serial, and tree configuration [5], [6]. Optimal distributed detection algorithms have been focused on optimality under the Neyman-Pearson and Bayesian detection criteria. Under the assumption of conditionally independent observations, the optimal fusion rules are given by likelihood ratio tests at the individual sensors and at the fusion center [7]. For conditionally dependent observations, the optimal fusion rules become intractable: they do not reduce to likelihood ratio tests [8], [9]. Recently, distributed detection algorithms have also been investigated under several communication-constraints [2], [10], [11]. Chamberland and

This work is supported by NSF under NSF CAREER award CCR-0105654.
Veeravalli [11] show that under certain conditions, for an $N$-sensor network with a capacity constraint of $N$ bits per time unit, having each sensor transmitting one bit is optimum. Thomopoulos and Zhang investigate the distributed detection in the case of non-ideal channels [12]. In [13], Duman and Salehi consider the distributed detection over multi access channels where the fusion center gathers the decisions from local sensors via a multi-access channel.

All the aforementioned algorithms assume that the sensor decision statistics, either quantized or at full precision, can be transmitted error-free to the fusion center. Even though this assumption is valid in traditional sensor networks such as radars and sonar, it is impractical in wireless sensor networks where wireless links are subject to fading and interference. Furthermore, due to bandwidth and energy constraints, the use of powerful error correction codes is not viable. Recently, Chen et al. introduced channel-based decision fusion for network of sensors linked with fading channels [14]. Assuming parallel configuration, the authors incorporate the effect of fading in the detection process, and derive optimal fusion rules and some alternative fusion rules based on diversity combining techniques. The performance of the proposed fusion rules is evaluated through simulations; however, the optimality of the decision rules at local sensors and at the fusion center, and optimal designs (e.g., thresholds) are not considered in [14]. Recently, Chen and Willet have sown that optimal local decisions that minimize the error probability at the fusion center becomes a likelihood-ratio test (LRT) under some particular constraints on the fusion rule [15].

In this paper, we investigate the performance of the parallel distributed detection in wireless sensor networks. Assuming flat fading channels between local sensor nodes and the fusion center, we propose a parallel distributed detection system based on the LR-test. We derive the global detection and false alarm probabilities for this system and then use these probabilities to design the best threshold sets according to Neyman-Person criterion. We consider both single-bit and multi-bit based decision strategies. Finally, using numerical analysis of the theoretical results, we illustrate the performance of the proposed distributed detection.

The organization of the paper is as follows: In Section II, we describe the distributed detection structure being considered. 


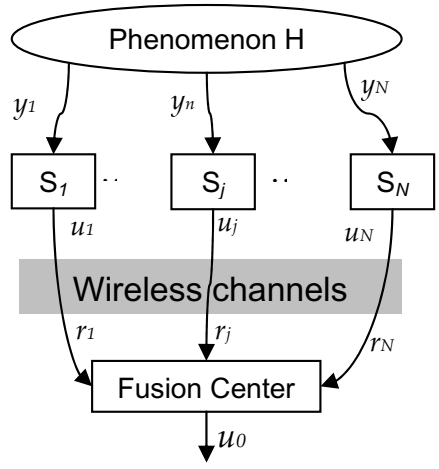

Fig. 1. Parallel configuration of a sensor system for distributed detection over fading channels

We derive the decision fusion rules and the relevant equations for false alarm and detection probabilities in Section III. The numerical examples are presented in Section IV and finally, the conclusions are summarized in Section V.

\section{System Model}

In Figure 1, we depict the block diagram of a parallel network of $N$ sensors for distributed detection of a binary hypothesis. The sensor node $S_{j}$ observes $y_{j}$ and makes a local binary decision denoted by $u_{j} \in\{0,1\}$. Using a binary modulation scheme, the local decisions are sent to the fusion center, $S_{0}$. Assuming frequency flat fading and additive noise, the signal received from $S_{j}$ is given by

$$
r_{j}=\sqrt{\rho} g_{j} s_{j}+n_{j}
$$

where $g_{j}$ is the complex-valued channel gain between $S_{j}$ and $S_{0}$, and $n_{j}$ is the additive noise for that link. Both $g_{j}$ and $n_{j} \sim$ $\mathcal{C N}(0,1)$, and they are independent and identically distributed for $j=1, \cdots, N$. The average energy of the transmitted signal $s_{j} \in\left\{s^{0}, s^{1}\right\}$ is normalized to unity so that $\rho$ is the expected signal-to-noise ratio (SNR) for each sensor node. We assume that channel state information (CSI), i.e., $\mathbf{g}=\left[g_{1}, \cdots, g_{N}\right]$, is known only at the fusion center.

The decision at the fusion center is based on the received signals, $\mathbf{r}=\left[r_{1}, r_{2}, \cdots, r_{N}\right]$. We assume that both the observations $u_{j}$ and the received signals $r_{j}$ are statistically independent for $j=1, \cdots, N$ conditioned on the hypothesis. The false alarm and detection probabilities at $S_{j}, j=0, \cdots, N$, are defined as $P_{F, j}=\operatorname{Pr}\left(u_{j}=1 \mid H_{0}\right), P_{D, j}=\operatorname{Pr}\left(u_{j}=\right.$ $\left.1 \mid H_{1}\right)$. Our goal is to derive fusion rules based on the NeymanPearson lemma, that is, for a prescribed bound on the global false alarm rate, $P_{F, 0}$, we wish to find the decision rules both at the local sensors and at the fusion center that maximize the global detection rate, $P_{D, 0}$.

\section{Distributed Detection for Fading Channels}

\section{A. Detection with Single-bit Local Decisions}

According to Neyman-Pearson lemma, the optimal decision rules at $S_{0}$ reduces to the likelihood ratio test

$$
\Gamma(\mathbf{r})=\frac{L\left(\mathbf{r} \mid H_{1}, \mathbf{g}\right)}{L\left(\mathbf{r} \mid H_{0}, \mathbf{g}\right)}=\prod_{j=1}^{N} \frac{p\left(r_{j} \mid H_{1}, g_{j}\right)}{p\left(r_{j} \mid H_{0}, g_{j}\right)} \underset{H_{0}}{\stackrel{H_{1}}{\gtrless}} t_{0}
$$

where $p\left(r_{j} \mid H_{1}, g_{j}\right)=P_{D, j} p_{n}\left(r_{j}^{(1)}\right)+\left(1-P_{D, j}\right) p_{n}\left(r_{j}^{(0)}\right)$, and $p\left(r_{j} \mid H_{0}, g_{j}\right)=P_{F, j} p_{n}\left(r_{j}^{(1)}\right)+\left(1-P_{F, j}\right) p_{n}\left(r_{j}^{(0)}\right)$, with $r_{j}^{(k)}=$ $r_{j}-\sqrt{\rho} g_{j} s^{k}$. Let $\Upsilon\left(r_{j}\right)=p\left(r_{j} \mid H_{1}, g_{j}\right) / p\left(r_{j} \mid H_{0}, g_{j}\right)$. With binary phase shift keying (BPSK) modulation, we can simplify $\Upsilon\left(r_{j}\right)$ to

$$
\Upsilon\left(r_{j}\right)=\frac{P_{D, j} \xi_{j}+1-P_{D, j}}{P_{F, j} \xi_{j}+1-P_{F, j}}
$$

where $\xi_{j}=\exp \left(4 \sqrt{\rho} \Re\left\{r_{j} g_{j}^{*}\right\}\right)$. Substituting (3) into (2), and taking the logarithm of both sides, we obtain the LR-test at the fusion center as

$$
\sum_{j=1}^{N} \Upsilon^{*}\left(r_{j}\right) \underset{H_{0}}{\stackrel{H_{1}}{\gtrless}} t_{0}^{*}
$$

where $\Upsilon^{*}\left(r_{j}\right)=\log \left(\Upsilon\left(r_{j}\right)\right)$, and $t_{0}^{*}=\log \left(t_{0}\right)$, and $t_{0}$ is a threshold to be determined. Although it is straightforward to implement the fusion rule described by (4), note that it requires (i) the exact knowledge of the channel gain $g_{j}$ and (ii) the false alarm \& detection probabilities at the local sensors. We assume quasistatic fading where the channel remains constant for a long period. In that case, the CSI can be estimated at the fusion center using a training sequence.

\section{False Alarm and Detection Probabilities}

In order to assess the performance of the decision fusion rule in (4), and to develop optimal thresholds, we need to derive the false alarm and detection probabilities. At the fusion center, the false alarm probability is given by

$$
P_{F, 0}=\operatorname{Pr}\left(\sum_{j=1}^{N} \Upsilon^{*}\left(r_{j}\right)>t_{0}^{*} \mid H_{0}\right) .
$$

Noting that we have $2^{N}$ different possible decision vectors $\mathbf{u}=\left[u_{1}, \cdots, u_{N}\right]$, and using the total probability theorem, we have

$P_{F, 0}=\sum_{k=0}^{2^{N}-1} \operatorname{Pr}\left(\mathbf{u}=\mathbf{u}_{k} \mid H_{0}\right) \operatorname{Pr}\left(\sum_{j=1}^{N} \Upsilon^{*}\left(r_{j}\right)>t_{0}^{*} \mid \mathbf{u}=\mathbf{u}_{k}, H_{0}\right)$

where $\operatorname{Pr}\left(\mathbf{u}=\mathbf{u}_{k} \mid H_{0}\right)=\prod_{i=1, \mathbf{u}=\mathbf{u}_{k}}^{N} P_{F, i}^{u_{i}}\left(1-P_{F, i}\right)^{1-u_{i}}, \mathbf{u}_{k}=$ $\operatorname{bin}(k, N)$, and $\operatorname{bin}(k, N)$ is the binary vector representation of $k$ using $N$ bits, e.g., $[0,0,1,1]=\operatorname{bin}(3,4)$. Substituting (3) into (6), using $r_{j}=\left(2 u_{j}-1\right) \sqrt{\rho} g_{j}+n_{j}$, and observing that $\operatorname{Pr}\left(\cdot \mid \mathbf{u}=\mathbf{u}_{k}, H_{0}\right)$ in (6) is independent of the underlying hypothesis $H_{0}$, we can express (6) as

$$
P_{F, 0}=\sum_{k=0}^{2^{N}-1} \operatorname{Pr}\left(\mathbf{u}=\mathbf{u}_{k} \mid H_{0}\right) \operatorname{Pr}\left(\sum_{j=1}^{N} \Upsilon_{u_{j}}^{*}\left(n_{j}^{\prime}\right)>t_{0}^{*} \mid \mathbf{u}=\mathbf{u}_{k}\right)
$$


where $\Upsilon_{u_{j}}^{*}\left(n_{j}^{\prime}\right)=\log \frac{P_{D, j} e^{\left(2 u_{j}-1\right) 4 \rho\left|g_{j}\right|^{2}+4 \sqrt{\rho} n_{j}^{\prime}}+1-P_{D, j}}{P_{F, j} e^{\left(2 u_{j}-1\right) 4 \rho\left|g_{j}\right|^{2}+4 \sqrt{\rho} n_{j}^{\prime}}+1-P_{F, j}}$, with $n_{j}^{\prime} \sim \mathcal{C}\left(0,\left|g_{j}\right|^{2} / 2\right)$. Let $\Gamma_{\mathbf{u}_{k}}^{*}=\sum_{j=1}^{N} \Upsilon_{u_{j}}^{*}\left(n_{j}^{\prime}\right)$, and denote the cumulative distribution of $\Gamma_{\mathbf{u}_{k}}^{*}$ by $F_{\Gamma_{\mathbf{u}_{k}}^{*}}(\cdot)$. Then we can rewrite (7) as

$$
P_{F, 0}=1-\sum_{k=0}^{2^{N}-1} \operatorname{Pr}\left(\mathbf{u}=\mathbf{u}_{k} \mid H_{0}\right) F_{\Gamma_{\mathbf{u}_{k}}^{*}}\left(t_{0}^{*}\right) .
$$

Using probability theory, we can show that the density and cumulative distribution of $\Upsilon_{u_{j}}^{*}\left(n_{j}^{\prime}\right)$ are given by (9) and (10), respectively, shown at the top of next page. Hence, using (9), (10), and the fact that $\Upsilon_{u_{j}}^{*}\left(n_{j}^{\prime}\right)$ are independent for $j=$ $1, \cdots, N$, we obtain $F_{\Upsilon_{u}^{*}}($.$) given in (11) at the next page,$ where $A_{k}=\sum_{j=1}^{k} \log \frac{1-P_{D, j}}{1-P_{F, j}}$ and $B_{k}=\sum_{j=1}^{k} \log \frac{P_{D, j}}{P_{F, j}}$. Using (11) and (8), with the knowledge of the detection and false alarm probabilities at the local sensors, we can finally evaluate the global false alarm probability, $P_{F, 0}$. In a similar fashion, the detection probability can be computed as

$$
P_{D, 0}=1-\sum_{k=0}^{2^{N}-1} \operatorname{Pr}\left(\mathbf{u}=\mathbf{u}_{k} \mid H_{1}\right) F_{\Gamma_{\mathbf{u}_{k}^{*}}^{*}}\left(t_{0}^{*}\right) .
$$

where $\operatorname{Pr}\left(\mathbf{u}=\mathbf{u}_{k} \mid H_{1}\right)=\prod_{i=1, \mathbf{u}=\mathbf{u}_{k}}^{N} P_{D, i}^{u_{i}}\left(1-P_{D, i}\right)^{1-u_{i}}$.

Although the fusion center uses a likelihood ratio test, determining the actual parameters for the best detection is a formidable task. This is because, according to NP-lemma, one needs to make an exhaustive search over all $P_{F, i}$ and $P_{D, i}$ to determine $t_{0}^{*}$ so that for some $P_{F, 0} \leq \alpha, P_{D, 0}$ is maximized. Since analytical solution is not tractable, the optimal solution can be found for only small values of $N$.

\section{Design Issues}

So far, we have not specified the detection rule at the local sensors. In the absence of errors in the transmissions from local sensors to the fusion center, it can be shown that the optimal tests at the local sensors are also LR-tests. If the links are subject to fading and noise, while the optimal fusion rule according to NP-lemma reduces to a likelihood-ratio test at the fusion center, the optimal tests at the local sensors are not easy to derive. However, one still needs some detection rule, and so, without claiming any optimality, we propose the use of LR-test also at the local sensors. The numerical examples in Section IV will justify that the detection in this fashion performs remarkably well.

The LR-test at the local sensors is given by

$$
\Lambda^{*}\left(y_{j}\right)=\log \frac{p\left(y_{j} \mid H_{1}\right)}{p\left(y_{j} \mid H_{0}\right)} \underset{H_{0}}{\stackrel{H_{1}}{\gtrless}} t_{j}^{*}
$$

Assume that the distribution of the observations $y_{j}$ is known, and let $F_{i}(\cdot)$ denote the cumulative distribution of $\Lambda^{*}\left(y_{j}\right)$ under the hypothesis $H_{i}, i=0,1$. Then, we can express the false alarm and detection probabilities at $S_{j}$ as $P_{F, j}=$ $1-F_{0}\left(t_{j}^{*}\right)$ and $P_{D, j}=1-F_{1}\left(t_{j}^{*}\right)$, respectively. Hence, the design of the detection rules reduces to the determination of the thresholds $t_{j}^{*}$ that maximize the $P_{D, 0}$ for some fixed $P_{F, 0}$.
As usual in distributed detection problems, an analytical solution is not feasible; therefore, we resort to numerical search procedures to determine the decision fusion rules. A simplistic approach is to set the thresholds at all sensor nodes the same, however, in that case, one can not guarantee the maximization of the global detection probability.

\section{B. Detection with Multi-bit Local Decisions}

Thus far, we allowed transmission of only one bit per sensor to the fusion center. Under fading and noisy channel assumptions, making multiple-bit decisions and sending more than one bit to the fusion center, although sacrificing from bandwidth and power, might improve the detection probability. In the sequel, we study a more general problem to find optimal number of the decision bits (i.e., number of quantization levels) at each sensor. Our goal is to determine the best (possibly) multiple-bit decision rules that provide the optimal power/performance tradeoff.

The above technique can readily be generalized to multi-bit case. Assume, for example, the $k^{\text {th }}$ sensor node generates a $b_{k}$-bit decision and employs $2^{b_{k}}$-ary modulation to transmit its decision to the fusion center. Let $\tau_{1}^{(k)}<\tau_{2}^{(k)} \ldots<\tau_{2^{b_{k}-1}}^{(k)}$ be thresholds at node $k$, and let the observation samples, $y_{k}, k=$ $1, \ldots, N$ are confined to the interval $\left[\tau_{\min }, \tau_{\max }\right]$. The signal $s_{l}^{(k)}$ is transmitted whenever $y_{k} \in\left(\tau_{l-1}^{(k)}, \tau_{l}^{(k)}\right], l=1, \ldots, 2^{b_{k}}$. Note that $\tau_{0}^{(k)}=\tau_{\min }$ and $\tau_{2^{b_{k}}}^{(k)}=\tau_{\max }$. For signals that take any value in the real line, we have $\tau_{\min }=-\infty$ and $\tau_{\max }=\infty$.

The likelihood ratio test can be expressed as

$$
\Lambda(\mathbf{r})=\sum_{k=1}^{N} \log \frac{\sum_{l=1}^{2^{b_{k}}} P_{k, l}^{(1)} p_{n}\left(r_{k}-\sqrt{\rho} g_{k} s_{l}^{(k)}\right)}{\sum_{l=1}^{2^{b_{k}}} P_{k, l}^{(0)} p_{n}\left(r_{k}-\sqrt{\rho} g_{k} s_{l}^{(k)}\right)}
$$

where $P_{k, l}^{(m)}=\operatorname{Pr}\left(y_{k} \in\left(\tau_{l-1}^{(k)}, \tau_{l}^{(k)}\right] \mid H_{m}\right)$. The false alarm and detection rates follow as

$$
\begin{aligned}
& P_{F, 0}=\operatorname{Pr}\left(\Gamma(\mathbf{r})>t_{0}^{*} \mid H_{0}\right) \\
& P_{D, 0}=\operatorname{Pr}\left(\Gamma(\mathbf{r})>t_{0}^{*} \mid H_{1}\right) .
\end{aligned}
$$

Finally, we can write the optimization problem for the best multiple bit decision rules as

$$
\begin{array}{ll}
\max & P_{D, 0} \\
\text { s.t. } & P_{F, 0} \leq \alpha \text { and } \sum_{k=1}^{N} b_{k}=N_{\text {total }} \\
& \tau_{\min } \leq \tau_{l}^{(k)} \leq \tau_{\max }, k=1, \ldots, N, l=1, \ldots, 2^{b_{k}} .
\end{array}
$$

The optimization in (18) is not a convex problem over the set of thresholds and the number of decision bits. Therefore, it is very difficult to solve the problem using standard optimization tools. Instead, one can employ exhaustive search methods to determine the optimal number of bits and the thresholds, which is a formidable task. A suboptimal method is to restrict the search over only the set of $b_{k}$ values satisfying the total bid budget constraint and using locally most optimal decision thresholds at each node for that $b_{k}$ value. 


$$
\begin{aligned}
& f_{\Upsilon_{u_{j}}^{*}}(y)=\frac{\left(P_{D, j}-P_{F, j}\right) e^{y}}{\left(P_{D, j}-P_{F, j} e^{y}\right)\left(\left(1-P_{F, j}\right) e^{y}-\left(1-P_{D, j}\right)\right)} \times \\
& \frac{1}{\sqrt{16 \pi \rho\left|g_{j}\right|^{2}}} \exp \left(-\left(\log \frac{\left(1-P_{F, j}\right) e^{y}-\left(1-P_{D, j}\right)}{P_{D, j}-P_{F, j} e^{y}}-\left(2 u_{j}-1\right) 4 \rho\left|g_{j}\right|^{2}\right)^{2} / 16 \rho\left|g_{j}\right|^{2}\right) \\
& F_{\Upsilon_{u_{j}}^{*}}(a)=\left\{\begin{array}{cl}
0 & a<\log \frac{1-P_{D, j}}{1-P_{F, j}} \\
Q\left(\left(\frac{\left(1-P_{F, j}\right) e^{a}-\left(1-P_{D, j}\right)}{P_{D, j}-P_{F, j} e^{a}}-4 \rho\left|g_{j}\right|^{2}\right) / 2\left|g_{j}\right| \sqrt{2 \rho}\right) & \log \frac{1-P_{D, j}}{1-P_{F} j}<a<\log \frac{P_{D, j}}{P_{F, j}} \\
1 & a>\log \frac{P_{D, j}}{P_{F, j}}
\end{array}\right. \\
& F_{\Gamma_{\mathbf{u}_{k}}^{*}}\left(t_{0}^{*}\right)=\int_{A_{N-1}}^{B_{N-1}} \cdots \int_{A_{1}}^{B_{1}} f_{\Upsilon_{u_{1}}^{*}}\left(y_{1}\right) \prod_{j=2}^{N-1} f_{\Upsilon_{u_{1}}^{*}}\left(y_{j}-y_{j-1}\right) F_{\Upsilon_{u_{N}}^{*}}\left(t_{0}^{*}-y_{N-1}\right) d y_{1} \cdots d y_{N-1}
\end{aligned}
$$

\section{NUMERICAL RESULTS}

In this section, we illustrate the performance of the parallel decision fusion using numerical simulations. We consider the detection of a DC signal in additive white Gaussian noise (AWGN), i.e., $y=m+n$, where $m=k$ if $H_{k}$ is correct for $k=0,1$, and $n \sim \mathcal{C}(0,1)$. The local sensors employ LRtests to perform detection and use BPSK modulated signals \pm 1 to transmit their decisions. By using the expressions (e.g., (8), (11), and (12)) developed in Section III-A, we obtain the optimal thresholds by exhaustive search. We used the numerical integration routine QUADL in MATLAB ${ }^{\circledR}$.

We first illustrate the performance of the parallel detection based on single-bit decisions. Figures 2.a and 2.b depict the receiver operating characteristics (ROC) for $\rho=1$ and $\rho=3$, respectively. Identical thresholds are assumed at local sensors. We compute the average probabilities of the false alarm and detection assuming Rayleigh fading channels. We observe that as number of sensor nodes increases, the detection performance improves significantly. However, for all cases in these figures, we observe that the performance degradation due to the noise channel is significant with respect to the performance of the centralized detection which assumes the availability of noise-free observations at the fusion center.

Next, we illustrate the optimal threshold design for the parallel decision fusion. We assume that the local sensors employ LR tests to perform detection. By using the expressions (e.g., (8)- (12)) developed in Section III-A, we obtain the optimal threshold values $t_{i}, i=1, \cdots, N$ by an exhaustive search. In Figure 2.c, we illustrate the ROC curves for parallel distributed detection with $N=2$ local sensors. The false alarm and detection probabilities are averaged over the channel distribution, e.g., $P_{F, 0}=E_{\mathbf{g}}\left\{P_{F, 0} \mid \mathbf{g}\right\}$. We consider 4 detection schemes: (i) ideal local detectors, i.e., $P_{D, j}=1, P_{F, j}=0$, (ii) LR tests at all sensors with different local thresholds, (iii) LR tests with the same local thresholds, and (iv) LR tests with all thresholds set to 0 , i.e., $t_{j}^{*}=0$. For the cases (ii) and (iii), we perform an exhaustive search to determine the best threshold sets. We observe that the performance with the first scheme is superior to the LR test based detection schemes. This is expected since in the first scheme, the errors associated with the distributed detection are introduced during the transmission of the local decisions. When the local detectors employ LR tests, the best detection probability is obtained when we do not put any constraint on the thresholds (Case (ii)) and perform the search over all possible threshold sets. This search is computationally very complex. If we limit the search such that each local sensor use the same threshold (Case (iii)), the computation burden is much less, but we observe some performance degradation. The performance is worst for the heuristic detection where threshold is set to 0 at each local sensor node (Case (iv)).

We finally illustrate an example for multi-bit decision schemes. In Figure 2.d, for a network of two sensor nodes, we compare the detection performance between three different schemes where a total of $N_{T}=2,3$ or 4 bits are allowed. Since the search for optimal thresholds is not practical, we use a fixed threshold scheme. Specifically, for the DC-level problem at hand, we set $\tau_{l}=m / 2+l \delta, l=-\left(2^{b_{k}-1}-\right.$ $1), \ldots, 0,1,\left(2^{b_{k}-1}-1\right)$, where $\delta=m / 2^{b_{k}}$ and $b_{k}$ denotes the number of bits for the decision at node $k$. We preferred to use this threshold scheme since this one provided the best detection performance among various other threshold schemes we simulated. Figure 2.d depicts the ROC curves for the case where the links between the local nodes and the fusion center have similar fading levels. For $N_{T}=2$, the only possible scheme is to make single-bit decisions at both sensor nodes. For $N_{T}=3$, we may have two cases: $\left(b_{1}, b_{2}\right)=(1,2)$ or $(2,1)$, and for $N_{T}=4$, we have $\left(b_{1}, b_{2}\right)=(1,3),(2,2)$, or $(3,1)$. From the figure, we observe that detection performance with single-bit decisions is superior to other schemes. For this case where the links have similar quality, it is seen that different bit assignment schemes provide similar quality for a given total bit budget. On the other hand, simulations indicate that if one of the link is severely fading, the best $P_{D, 0}$ for a fixed $N_{T}$ is attained when more bits are assigned for the sensor node whose fading gain is smaller [16]. 

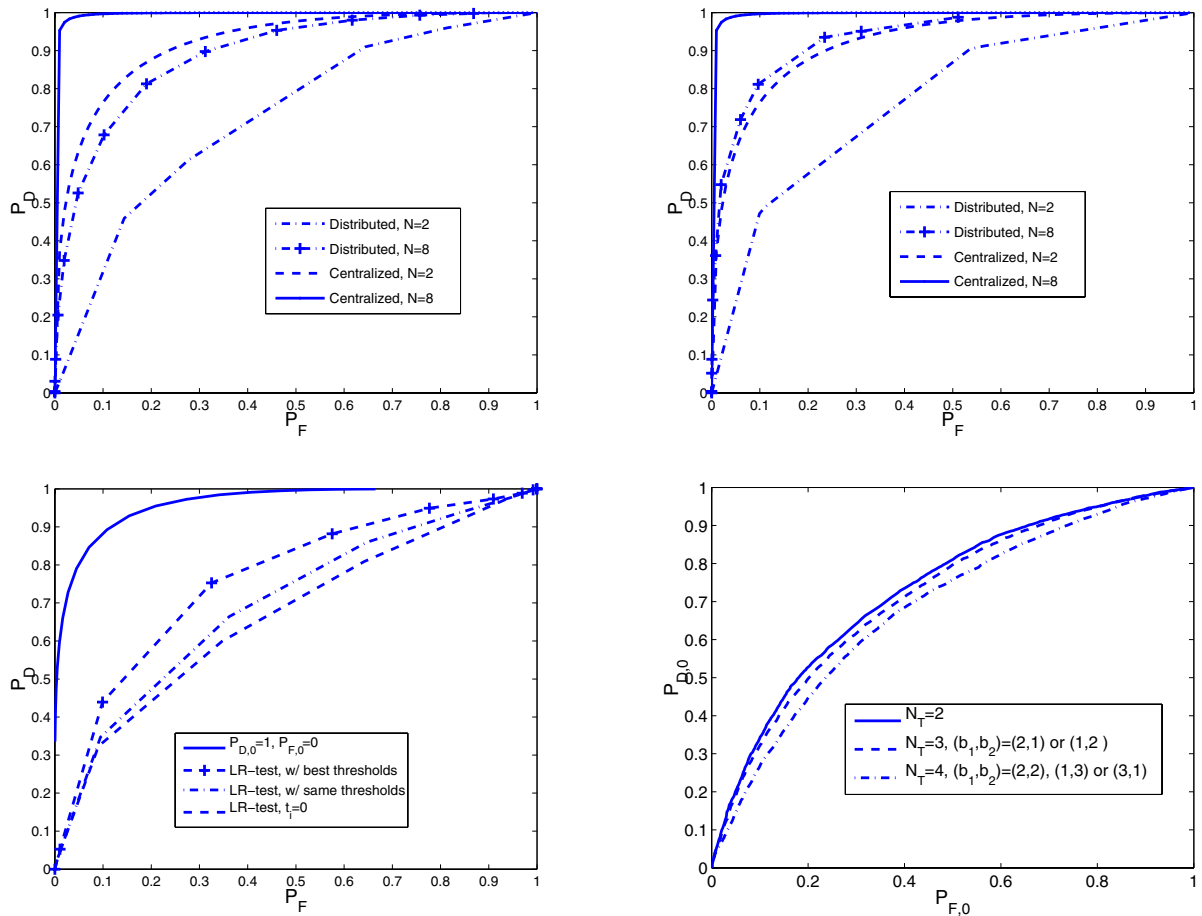

Fig. 2. ROC-curves for DC-level detection problem using parallel networks. Simulation parameters: DC-level $m=1, N=2$ or 8 sensors, (a) (Upper left) $\rho=0 \mathrm{~dB}$ and (b) (Upper right) $\rho=4.77 \mathrm{~dB}$, Rayleigh fading channel. (c) (Lower left) Optimal threshold design for $N=2, \rho=0 \mathrm{~dB}$ (d) (Lower right) performance with multi-bit local decisions, $N=2, \rho=4.77 \mathrm{~dB}$.

\section{CONCLUSION}

We investigated the performance of parallel distributed detection in WSN under the assumption of fading channels. We studied the likelihood ratio based fusion rule that incorporates fading in the distributed detection problem, derived the global false alarm and detection probabilities and used them in determining the thresholds at the local sensors and the fusion center using the Neyman-Pearson criterion. We also investigated the performance for multi-bit decision strategies. Finally, through numerical analysis and simulations, we designed several parallel fusion rules and evaluated their performance. Simulations indicate that parallel detection in wireless sensor networks performs well and in many cases, making single-bit local decisions provides superior detection rates than making multi-bit decisions.

\section{REFERENCES}

[1] Y.Sankarasubramaniam I.F.Akyildiz, W.Su and E.Cayirci, "A survey on sensor networks," IEEE Communications Magazine, pp. 102-114, August 2002.

[2] J.Kusuma S.S.Pradhan and K.Ramchandran, "Distributed compression in a dense microsensor network," IEEE Signal Processing Magazine, vol. 19, no. 2, pp. 51-60, March 2002.

[3] Y.H.Hu D.Li, K.D.Wang and A.M.Sayeed, "Detection, classfication and tracking of targets," IEEE Signal Processing Magazine, vol. 19, no. 2, pp. 17-29, March 2002.

[4] K.Yao J.C.Chen and R.E.Hudson, "Source localization and beamforming,” IEEE Signal Processing Magazine, vol. 19, no. 2, pp. 30-39, March 2002.
[5] R.Viswanathan and P.K.Varshney, "Distributed detection with multiple sensors-part i: Fundamentals," Proceedings of IEEE, vol. 85, no. 1, pp. 54-63, Jaunary 1997.

[6] S.A.Kassam R.S.Blum and H.V.Poor, "Distributed detection with multiple sensors: Part ii - advanced topics," Proceedings of the IEEE, vol. 85, no. 1, pp. 64-79, January 1997.

[7] P.K.Varshney, Distributed Detection and Data Fusion, Springer-Verlag, 1996.

[8] G. S. Lauer and N. R. Sandell, "Distributed detection of known signal in correlated noise," Tech. Rep., ALPHATECH, Burlington, MA, Mar. 1982.

[9] E. Drakopoulos and C. C. Lee, "Optimal multisensor fusion of correlated local decisions," IEEE Trans. Aerospace Elect. Syst., vol. 27, pp. 593 605, July 1991

[10] T.D.Lookabaugh M.Longo and R.M. Gray, "Quantization for decentralized hypothesis testing under communication constraints," IEEE Trans. on Inform. Theory, vol. 36, no. 2, pp. 241-255, March 1990.

[11] J.Chamberland and V.Veeravalli, "Decentralized detection in sensor networks," IEEE Transactions on Signal Processing, vol. 51, no. 2 , pp. 407-416, February 2003.

[12] S. C. Thomopoulos and L. Zhang, "Distributed decision fusion with networking delays and channel errors," Inform. Sci., vol. 66, pp. 91118, Dec. 1992

[13] T. M. Duman and M. Salehi, "Decentralized detection over multiple access channels," IEEE Trans. Aerospace Elect. Syst., vol. 34, pp. 469476, Apr. 1998

[14] B. Chen, R. Jiang, T. Kasetkasem, and P. K. Varshney, "Channel aware distributed detection in wireless sensor networks," IEEE Trans. Signal Processing, vol. 52, no. 12, pp. 3454-3458, Dec 2004.

[15] B. Chen and P. K. Willet, "On the optimality of the likelihood-ratio test for local sensor decision rules in the presence of nonideal channels," IEEE Transactions on Information Theory, vol. 51, no. 2, pp. 693-699, Feb 2005.

[16] Israfil Bahceci, Multiple-Input Multiple-Output Wireless Systems: Coding, Distributed Detection and Antenna Selection, Ph.D. thesis, Georgia Inst. of Tech., Dec 2005. 